\title{
Equilibrium shape of the aqueous humor-vitreous substitute interface in vitrectomized eyes
}

Krystyna Isakova ${ }^{1}$, Jan O. Pralits ${ }^{2}$, Mario R. Romano ${ }^{3}$, Jan-Willem M. Beenakker ${ }^{4}$, Denis P. Shamonin ${ }^{5}$, Rodolfo Repetto ${ }^{2}$

${ }^{1}$ Department of Mechanical, Materials and Manufacturing Engineering, University of Nottingham, Nottingham, UK, ${ }^{2}$ Department of Civil, Chemical and Environmental Engineering, University of Genoa, Italy, ${ }^{3}$ Department of Neurosciences, University of Naples Federico II, Naples, Italy, ${ }^{4}$ Department of Radiology, C.J. Gorter Center for High-Field MRI, Leiden University Medical Center, Leiden, The Netherlands and Department of Ophthalmology, Leiden University Medical Center, Leiden, The Netherlands, ${ }^{5}$ Department of Radiology, Division of Image Processing, Leiden University Medical Center, Leiden, The Netherlands

\section{Abstract}

Purpose: To predict the shape of the interface between aqueous humor and a gas or silicone oil (SO) tamponade in vitrectomized eyes. To quantify the tamponated retinal surface for various eye shapes, from emmetropic to highly myopic eyes.

Methods: We use a mathematical model to determine the equilibrium shape of the interface between the two fluids. The model is based on the volume of fluids (VOF) method. The governing equations are solved numerically using the free software OpenFOAM. We apply the model to the case of idealized, yet realistic, geometries of emmetropic and myopic eyes, as well as to the real geometry of the vitreous chamber reconstructed from magnetic resonance imaging (MRI) images.

Results: The numerical model allows us to compute the equilibrium shape of the interface between the aqueous humor and the tamponade fluid. From this we can compute the portion of the retinal surface that is effectively tamponated by the fluid. We compare the tamponating ability of gases and SOs. We also compare the tamponating effect in emmetropic and myopic eyes by computing both tamponated area and angular coverage.

Correspondence: Department of Civil, Chemical and Environmental Engineering, University of Genoa, Via Montallegro 1, 16145 Genoa, Italy.

E-mail: rodolfo.repetto@unige.it 
Conclusion: The numerical results show that gases have better tamponating properties than SOs. We also show that, in the case of SO, for a given filling ratio the percentage of tamponated retinal surface area is smaller in myopic eyes. The method is valuable for clinical purposes, especially in patients with pathological eye shapes, to predict the area of the retina that will be tamponated for a given amount of injected fluid.

Keywords: vitrectomy, tamponade fluids, surface tension, interface

\section{Introduction}

Rhegmatogenous retinal detachment (RRD) is the most common type of retinal detachment. It classically involves the presence of three factors: vitreous traction on the retinal surface, full-thickness retinal breaks, and liquefied vitreous allowing the passage of fluid from the preretinal space through the retinal breaks (RBs) into the subretinal space. ${ }^{1}$ The intraocular surgical approach to RRD is vitrectomy, aimed at relieving vitreous traction on the edge of the RBs. ${ }^{2}$ In order to close the passage between preretinal and subretinal spaces, it is necessary to induce reattachment between the retina and choroid. Intraocular tamponades, used during vitreoretinal surgery, aim at facilitating this reattachment, keeping in contact retina and choroid in correspondence of the RBs, until a chorio-retinal scar is well formed. ${ }^{3}$ In the early postoperative period, the patient has to maintain a precise head position as to maintain the tamponade fluid in contact with the RBs. ${ }^{4}$

Owing to the hydrophobic properties of tamponade fluids, after vitrectomy a pocket of aqueous humor is invariably present in the vitreous chamber. The maximum filling that the surgeon can normally obtain is approximately $90 \%$ of the volume of the vitreous chamber. ${ }^{5}$ For a given volume of tamponade fluid injected in the eye, the tamponated retinal surface is strongly affected by the shape of the interface between the tamponade fluid and the aqueous humor. The equilibrium configuration of the interface depends on the shape of the domain, on the physical properties of the two fluids (in particular on density difference, surface tension, and contact angle with the retina), and head orientation.

This problem was studied by Eames et al., ${ }^{6}$ who modeled the vitreous chamber as a sphere filled with two different fluids, and determined the shape of the interface using a mathematical approach as well as experiments on a model. From their analysis, the authors obtained a relationship between retinal coverage and the volume of tamponade fluid. The main assumption underlying the work by Eames et $a .^{6}$ is related to the use of a spherical eye model. In reality, the vitreous chamber is not spherical, in particular owing to the indentation produced in the front part by the lens. The concavity change of the domain in the anterior part is likely to significantly affect the shape of the interface; therefore, it is of clinical interest to study the equilibrium configuration of a tamponade fluid in a realistic eye geometry. In addition, understanding how the interface shape might change in myopic eyes, which are at a higher risk of developing 
retinal detachment and whose shape is different with respect to that of emmetropic eyes, is highly relevant. To increase the success rate of the surgery, it is important to predict the surface of the retina that will be effectively tamponated, which depends on the shape of the interface between the aqueous and the tamponade.

In this paper, we compute the shape of such an interface for both emmetropic and myopic eyes. We first consider idealized but realistic shapes of the vitreous chamber and, subsequently, real eye geometry reconstructed from MR-measurements. We focus our attention on cases in which the patient maintains the upright position, but the method could be applied to other cases without additional difficulties.

\section{Materials and methods}

\subsection{Mechanical properties of tamponade fluids}

It is well known that the shape of the static equilibrium interface between two fluids can be obtained by solving the Laplace-Young equation, imposing the contact angle at the triple line given by the intersection between the interface and the wall. The solution depends on the fluid densities, the surface tension at the interface, and the static contact angle at the solid wall. We note that fluid viscosity does not affect the equilibrium configuration of the interface. ${ }^{7}$ In this paper we consider two tamponades that are commonly used in vitrectomy: SO and gas. In Table 1 we list the relevant mechanical properties of these fluids and the corresponding references. The properties of the SO are those of Oxane 1300. The surface tension and contact angle for the gas are taken from Eames et al. ${ }^{6}$, while the density is that of air. The density of a tamponade gas depends, in general, on its chemical compound (C2F6, C3F8, or SF6) and concentration, but is nevertheless much smaller than that of the aqueous humor. Therefore, we believe that our choice of gas density does not make any tangible difference in terms of interface shape. ${ }^{6}$ We note that the density of SO is much greater than that of gas. Also, the contact angle between SO, aqueous, and retina is significantly smaller than in the case of the interface between gas and aqueous. Thus, we expect the interface shape in the two cases to be substantially different.

Table 1. Mechanical properties of fluids. Density and surface tension properties are taken from Eames et $a l^{6}{ }^{6}$ The contact angle values were obtained by averaging the values proposed by Joussen, Wong ${ }^{8}$ and Fawcett et al. ${ }^{5}$ (See also Table 1 in Eames et al. ${ }^{6}$ )

\begin{tabular}{|l|l|l|l|}
\hline & Density $\left(\mathbf{k g} / \mathbf{m}^{3}\right)$ & $\begin{array}{l}\text { Surface tension } \\
\text { with aqueous }(\mathbf{N} / \mathbf{m})\end{array}$ & $\begin{array}{l}\text { Contact angle with } \\
\text { the retina (deg) }\end{array}$ \\
\hline $\begin{array}{l}\text { SO } \\
\text { (Oxane } \\
\text { 1300) }\end{array}$ & 980 & 0.044 & $16.17 \pm 1.23$ \\
\hline Gas & 1.225 & 0.07 & $30.74 \pm 4.24$ \\
\hline $\begin{array}{l}\text { Aqueous } \\
\text { humor }\end{array}$ & 1000 & - & - \\
\hline
\end{tabular}




\subsection{Description of the model}

The shape of the interface is computed numerically using the VOF method. The basic principle of this method is to introduce an additional function that provides the volume fraction of the tracked phase in the computational cell. This function is equal to one when the cell is entirely occupied by one fluid, vanishes when it is entirely occupied by the other fluid, and assumes an intermediate value when the cell contains the interface. An additional equation for such a function is introduced, which imposes that this function moves together with the fluid (its material derivative is equal to zero). The VOF method is known to provide a simple and economical way of tracking an interface between two fluids in 3D. ${ }^{9,10}$ Numerical simulations are performed using the free software OpenFOAM. ${ }^{11}$ All meshes are generated using the snappyHexMesh tool by OpenFOAM, which produces unstructured meshes consisting of tetragonal and hexahedral volumes. We perform fully 3D simulations using meshes consisting on average of 1.7 million volumes, and run the code in parallel on a 32processor computer and, for some cases, at the HPC center CINECA, in Italy. Careful mesh-independence tests have been carried out for all simulations. The numerical simulations are run by fixing the volume ratio (ratio of the volume of the injected tamponade fluid to the total volume of the domain) and setting an initially flat and horizontal shape of the interface. Advancing in time, the interface evolves towards its equilibrium shape; when a steady solution is obtained, the simulation ends. As noted in the previous section, viscosity does not affect the final configuration reached by the interface. However, it obviously affects the transient phase of the computation before a steady state is reached. If the viscosity of the two fluids is very large, convergence is obtained over long times. On the other hand, in the case of low viscosity, waves may form on the interface that can lead to numerical instabilities. In the course of the simulations, the values of the viscosity of the two fluids were tuned in order to optimize numerical efficiency.

\subsection{Model geometry}

\subsubsection{Idealized realistic eye shapes}

We first consider idealized, yet realistic shapes of the vitreous chamber in phakic eyes. The geometry is constructed on the basis of data from Atchison et al. ${ }^{12}$ The resulting vitreous chamber domain for an emmetropic eye is shown in Figure 1a on a vertical cross-section of symmetry. The domain is axisymmetric with respect to the horizontal axis that passes through the center of the lens. Geometries representing myopic eyes are constructed using data reported by Atchison et al. ${ }^{13}$ (see Table 1 of their paper), by stretching the emmetropic eye shape in all directions. However, for simplicity, we maintain the domain axisymmetric, thus imposing that the inferior-superior and nasal-temporal lengths of the vitreous chamber are equal to each other (averaging the values reported by Atchison et $a .^{13}$ for such lengths). In fact, according to Table 1 in the authors' paper, the differences between these two lengths are very small. 


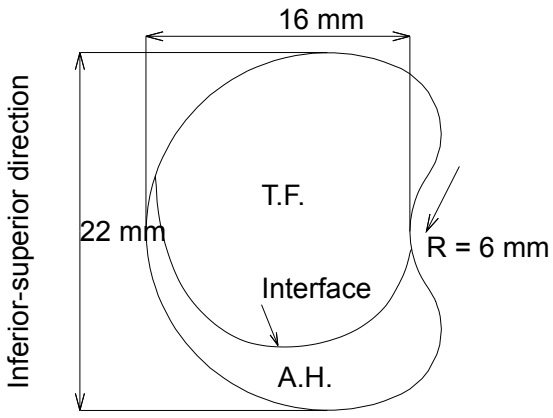

(a)
Anterior-posterior direction

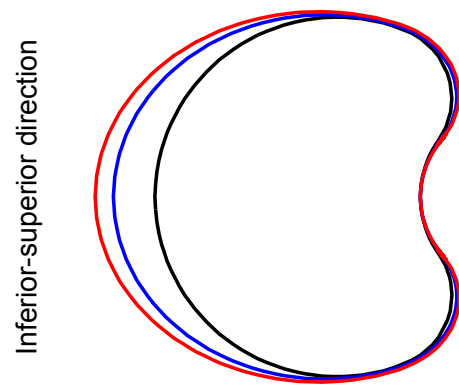

(b)

Anterior-posterior direction

Fig. 1. (a) Vertical symmetry cross-section of the domain for an emmetropic eye. The domain is filled with two immiscible fluids: the tamponade fluid (T.F.) and the aqueous humor (A.H.). All geometrical measurements are taken from Atchison et $a .^{12}(\mathrm{~b})$ Vertical cross-sections of myopic eyes. The domains have been obtained by stretching the shape of the emmetropic eye in all directions, according to Atchison et al. ${ }^{13}$ In the figure, the black line corresponds to the normal eye; blue line refers to myopic eyes with an axial length of $25.5 \mathrm{~mm}$, height and width of 22.3 $\mathrm{mm}$; and red line to an axial length of $26.6 \mathrm{~mm}$, height and width of $22.85 \mathrm{~mm}$.

Various myopic eye shapes reconstructed in this way are shown in Figure 1b, corresponding to different axial lengths of the eye, where, following Atchison et al., ${ }^{13}$ the axial length is defined as the distance between the anterior cornea and retinal pole. Obviously, real eyes show far greater complexity in shapes than what is considered in this section. However, adopting idealized yet realistic geometries allows us to draw general conclusions. The case of a real eye shape is also considered in this work, with the purpose of showing that the present mathematical model is also applicable in such cases.

\subsubsection{Real reconstructed eye shapes}

Retinal contours of real eyes were obtained from high-resolution MR-images, which were semi-automatically segmented, as described by Beenakker et al..$^{14,15}$ In short, ocular MR-measurements were performed on a Philips Achieva 7 Tesla (Best, The Netherlands) whole body magnet using a custom-made receive eye coil. ${ }^{14}$ The images were acquired using a 3D inversion recovery turbo gradient echo technique with an inversion time of $1280 \mathrm{~ms}$, a shot interval of $3 \mathrm{~s}$, and a turbo field echo factor of 92. The MR sequence parameters for repetition time (TR)/echo time (TE)/flip angles were: $2.5 \mathrm{~ms} / 4.55 \mathrm{~ms} / 16^{\circ}$. A cued-blinking protocol was used to minimize eye-motion artifacts. ${ }^{16}$ The scan resulted in a spatial resolution of $0.5 \times 1.0 \mathrm{~mm}^{3}$ and the scan time was slightly less than three minutes.

The MR-images were subsequently segmented semi-automatically by in-house developed software, based on the rapid-prototyping platform MevisLab (Fraunhofer 


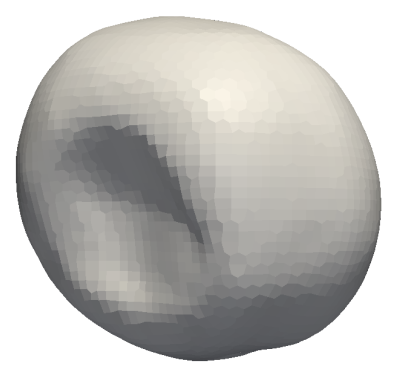

Fig. 2. Reconstructed shape of the vitreous body of an emmetropic eye from MR-images.

MeVis, Bremen, Germany). The central axis, the MR equivalent of the optical axis, was defined as the line from the center of the lens to the center of the vitreous body. A previous study on the reliability of the technique showed good agreement with partial coherence interferometry with a mean difference of $0.08 \mathrm{~mm}$ between the segmented MR-data and biometry. ${ }^{15}$ The study protocol was performed in accordance with the Declaration of Helsinki and was approved by the medical ethical committee of the Leiden University Medical Center. Informed consent was obtained from all participants.

\subsection{Model validation}

In order to validate the numerical model, we compared the numerical results with predictions of an in-house written code able to predict the shape of the interface between two fluids within a spherical domain. This is the problem considered in Eames et $a l .{ }^{6}$ Owing to the axisymmetry of the sphere, the problem reduces to compute the shape of a curve, which is the intersection of the interface with a vertical plane across the axis of symmetry.

Mathematically this problem is governed by a system of ordinary differential equations derived from the Laplace-Young law. ${ }^{7}$ The Laplace-Young equation is given by:

$$
2 k_{m}=\frac{\Delta \rho g}{\gamma} x_{I}+B
$$

where $k_{m}$ is the local mean curvature of the interface between the two fluids, $\Delta \rho=$ $\rho_{2}-\rho_{1}$ is the density difference, $g$ is the acceleration of gravity, $\gamma$ is the surface tension between two fluids, and $x_{I}(y)$ is the function describing the shape of the interface (see Fig. 3 for the coordinate system). Finally, $B$ is a constant that, adopting the system of coordinates depicted in Figure 3, represents the mean curvature at the origin. The local mean curvature of the interface can be evaluated as:

$$
2 k_{m}=-\nabla \cdot \mathbf{n},
$$

where $\mathbf{n}$ is the normal to the surface. 


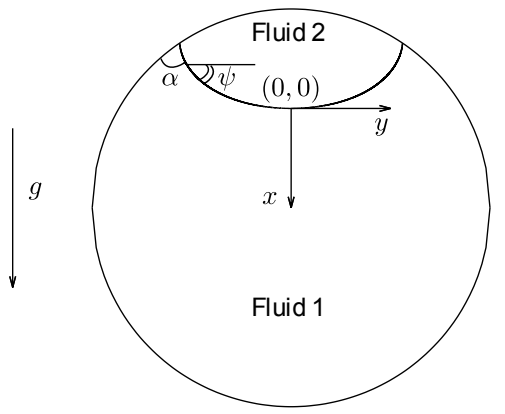

Fig. 3. Cross-section of the spherical domain with two superposed fluids. The origin is placed in the lowest point of the interface between fluids. The governing system of ordinary differential equations is given in terms of the slope angle $\psi$ in the interval $[0, \alpha]$, with $\alpha$ being a contact angle.

(a)

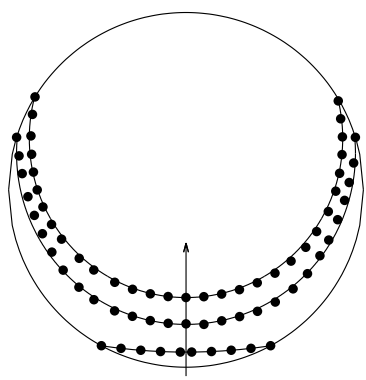

(b)

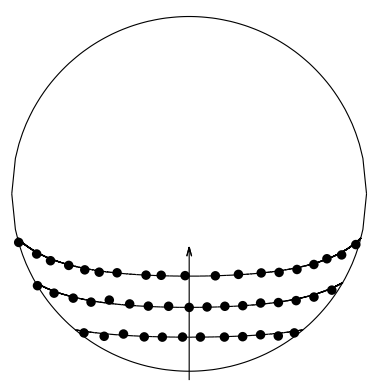

Fig. 4. Equilibrium shape of the interface in the spherical domain from the analytical solution (solid lines) and from the numerical model (dots) for different volume fractions of tamponade fluid $\left(V_{T . F .} / V=0.65,0.7,0.8\right)$. (a) SO, (b) gas. The arrow indicates decreasing values of $V_{T . F .} / V$. 
Since the function $x_{I}(y)$ can assume two values for a given $y$, it is convenient to use a parametric representation of the function, defining the position of the interface in terms of the slope angle $\psi$ shown in Figure 3, so that we can write:

$$
x=x(\psi), \quad y=y(\psi) .
$$

It is easy to show that the problem is governed by the following system of two ordinary differential equations:

$$
\begin{aligned}
& \frac{d x}{d \psi}=\frac{\sin \psi}{Q}, \\
& \frac{d y}{d \psi}=-\frac{\cos \psi}{Q},
\end{aligned}
$$

with $Q=\frac{\sin \psi}{y}-\frac{\Delta \rho}{\gamma} x-B$, which we solve subjected to the boundary conditions:

$$
x(0)=0 ; \quad y(0)=0 .
$$

The value of the mean curvature at the origin $B$ is unknown; thus, we iterate the integration of the above equations until the value of $\psi$ in correspondence of the wall equals the sought value of the contact angle. The iteration procedure is based on the bisection method.

In Figure 4 we plot the shape of the interface on a cross-section of the sphere. The radius of the sphere is equal to $0.01 \mathrm{~m}$. The solid line is obtained with the axisymmetric numerical model described above, whereas dots are relative to the fully $3 \mathrm{D}$ numerical solution obtained with the VOF method. Figure 4a refers to the case of aqueous-SO interface and Figure $4 \mathrm{~b}$ to the gas-aqueous interface. In both cases, the fluid below the interface is aqueous humor. The different curves correspond to different volume ratios of the tamponade fluid. In all cases the results from the two approaches are in very good agreement. The maximum difference between the fully $3 \mathrm{D}$ solution and the numerical model, normalized with the sphere radius, is $\approx 5 \times 10$ ${ }^{-5}$. This confirms the suitability of the VOF method for the purposes of this work.

\section{Results}

\subsection{Emmetropic eyes}

We first consider the case of emmetropic eyes and refer to the idealized eye shape reported in Figure 1a. Various equilibrium configurations of the interface are shown in Figure 5.

Each curve corresponds to a different degree of filling of the vitreous chamber by the tamponade fluid. Figures $5 \mathrm{a}$ and $\mathrm{b}$ are relative to the case of $\mathrm{SO}$, while Figures $5 \mathrm{c}$ and $d$ to the case of gas. In Figure 6 we also show 3D views of the interface, obtained with a filling ratio $V_{T . F .} / V=0.85$, where $V_{T . F}$. denotes the volume of the tamponade fluid and $V$ the total volume of the vitreous chamber. 


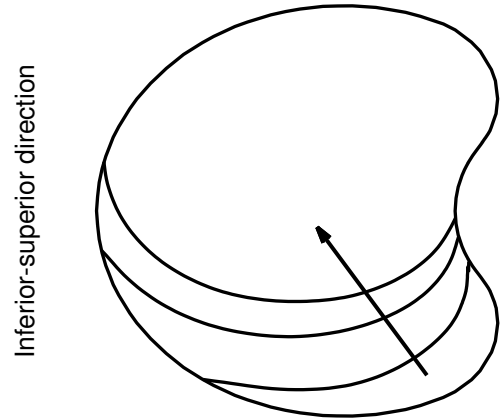

(a)

Posterior-anterior direction

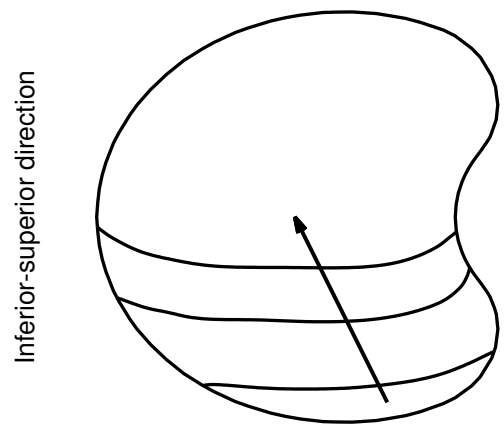

(c)
Posterior-anterior direction

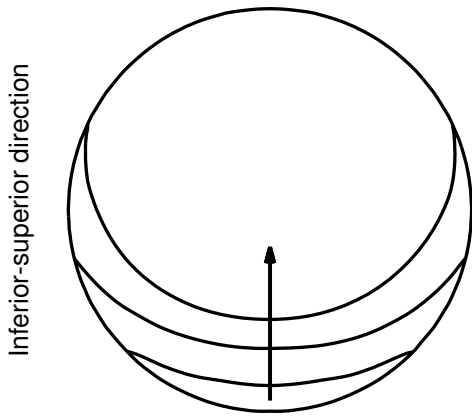

Nasal-temporal direction (b)

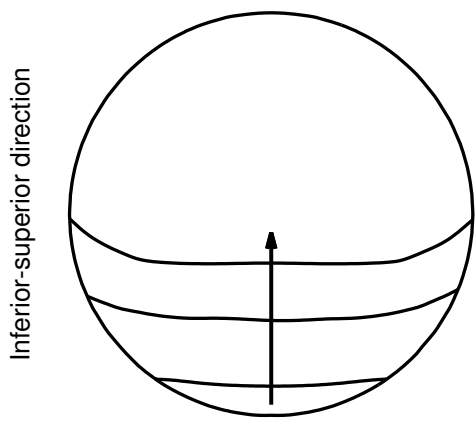

(d)

\section{Nasal-temporal direction}

Fig. 5. Equilibrium shape of the interface in the normal eye for the $S O(a, b)$ and gas $(c, d)$. For both cases we consider three different degrees of filling $\left(V_{T . F .} / V=0.60,0.75,0.90\right)$. The arrows indicate decreasing values of $V_{T . F .} / V$.
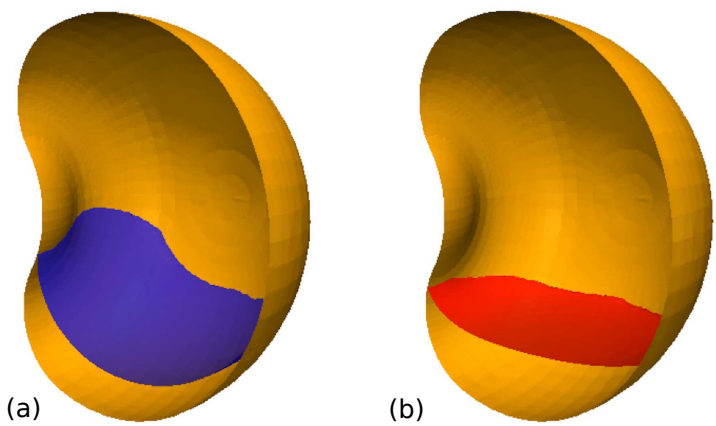

Fig. 6. Three-dimensional views of the interface shape: (a) $\mathrm{SO}$ and (b) gas. The degree of filling $V_{\text {T.F. }} / V$ is 0.85 in both cases. 
(a)

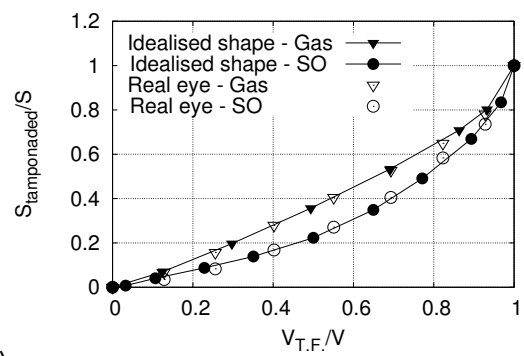

(b)

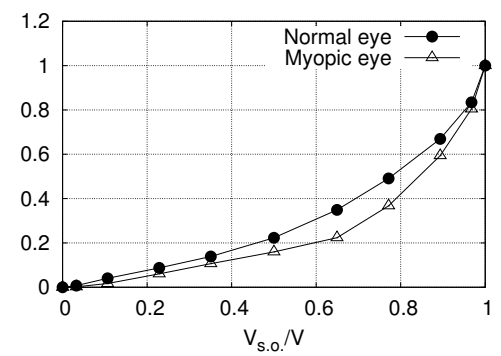

Fig. 7. (a) Relative tamponated surface as a function of the volume fraction for the case of SO and gas. Solid symbols refer to the idealized shapes of the vitreous chamber and open symbols to a real, reconstructed eye. (b) Relative tamponated surface as a function of the volume fraction for an emmetropic and a highly myopic eye, in the case of SO. The axial length of the myopic eye is equal to $24.6 \mathrm{~mm}$.

In Figure 6 we report the equilibrium shape of the interface for both SO (a) and intraocular gas (b). The equilibrium shape of the interface is significantly different with respect to that in a sphere. This is most evident looking at the anterior-posterior cross-sections (Figs. 5a,c). The indentation produced by the lens induces a change in the concavity of the domain and has a strong effect on the interface shape. Specifically, the elevation reached by the interface in the front and back regions of the domain can be very different. Interestingly enough, the contact line is higher in the front of the eye than in the back for high degrees of filling, whereas the opposite happens when the filling degree is small (see in particular Fig. 5a). Comparing the case of SO to that of gas (Figs. 5a,b vs Figs. 5c,d), it appears that the interface is flatter in the case of gas; thus, gas has better tamponating properties than the SO. This is clearly shown in Figure 7 a, where we plot the relative tamponated surface (i.e., the ratio between the surface area in contact with the tamponade fluid $S_{\text {tamponated }}$ and the total bounding surface of the vitreous chamber $S$ ) as a function of the volume fraction $V_{T \text {.F. }} / V$.

The two curves refer to SO and gas, respectively. The curve corresponding to gas is invariably higher than that corresponding to SO. This implies that, for a given amount of tamponade fluid injected into the eye (a given ratio $V_{T . F}$. $/ V$ ), the amount of surface tamponated by the gas is greater than that tamponated by the SO.

In Figure 8 we show the interface shape obtained in the case of a real emmetropic vitreous chamber reconstructed from MR-images, the geometry of which is shown in Figure 2. The degrees of filling correspond to those shown in Figure 5. In Figure 7a we also report, with open symbols, the results obtained for the case of the real eye. The model predictions for the idealized and real geometries are very close to one another. Also, in the case of the real geometry, the curves corresponding to SO and gas are clearly separated, with that relative to gas showing a larger amount of tamponated surface compared to SO. The results obviously depend on the specific eye chosen, and should be expected to change from subject to subject. However, the good match 
between the real and idealized cases shown in Figure 8a indicates that the model is also robust when used in complex geometries.

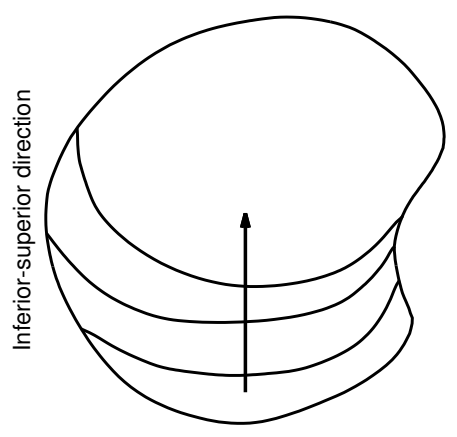

(a)

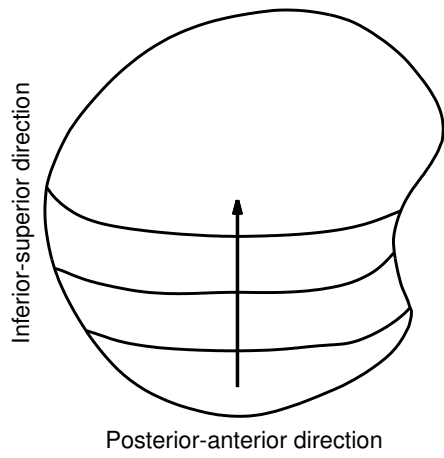

(b)

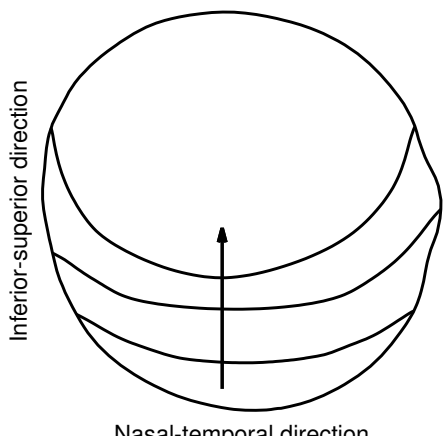

Nasal-temporal direction

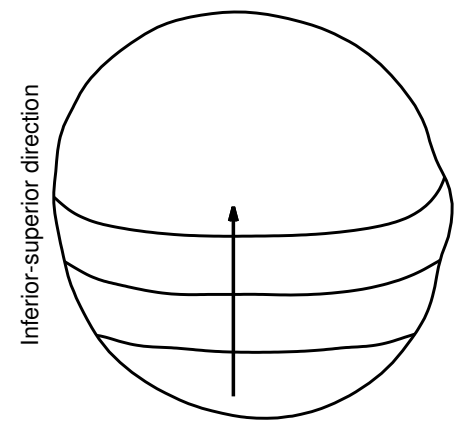

Nasal-temporal direction

Fig. 8. Equilibrium shapes of the interface in the real eye domain for the $\mathrm{SO}(a, b)$ and gas $(c, d)$. The degrees of filling are $V_{T . F .} / V=0.6,0.75,0.9$. The arrows indicate decreasing values of $V_{T . F .} / V$.

In addition to the relative tamponated area, we also calculated the angular coverage of the retina. In this case we only considered the surface of the vitreous chamber covered by the retina. Following clinical practice, this is defined as the region posterior to a plane parallel to the equator of the eye and at a distance of $6 \mathrm{~mm}$ from the limbus in the antero-posterior direction (Figs. 1 and 9a). We then introduce the angles $\Psi$ and $\Phi$, shown in Figure 9, defined as follows: the angle $\Psi$ is the angle of retinal coverage along an antero-posterior plane orthogonal to the equator (Fig. 9a); the angle $\Phi$ is the retinal coverage along the equatorial plane (Fig. 9b).

Comparisons of the tamponating properties of SOs and gases in terms of these angles are shown in Figure 10. Again, it appears that gas has better tamponating properties than SO. 


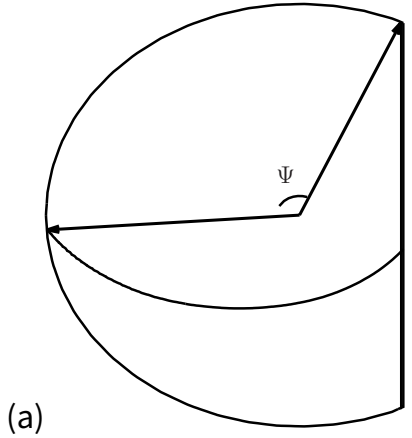

(b)

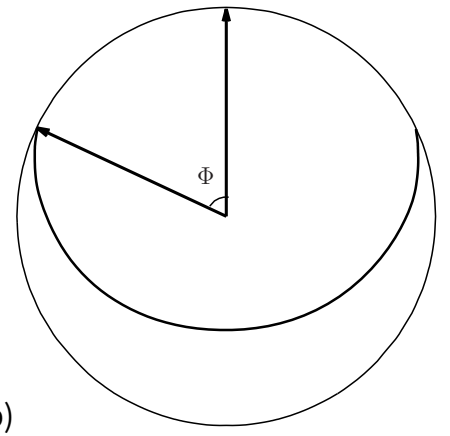

Fig. 9. Coverage angles $\Psi$ and $\Phi$. In (a) we show the way we measure the coverage angle $\Psi$ on the antero-posterior cross-section; in (b), the angle $\Phi$ on the equatorial plane.

(a)

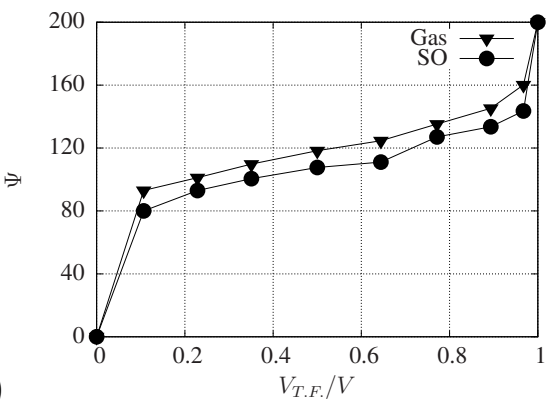

(b)

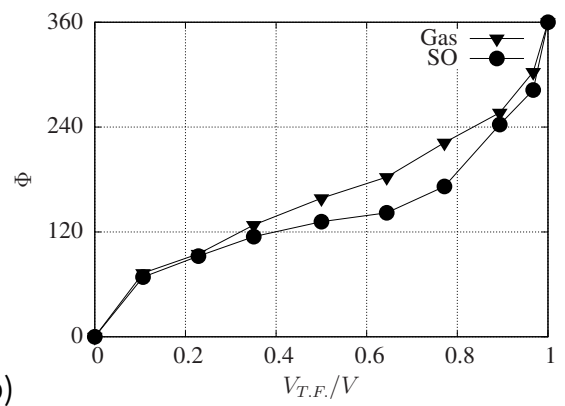

Fig. 10. Coverage angles $\Psi(a)$ and $\Phi(b)$ in degrees vs the filling ratio $V_{T . F .} / V$ in the case of SO and gas.

\subsection{Myopic eyes}

We now show the results obtained in the case of myopic eyes and compare them to those found in emmetropic eyes. In Figure 11 we plot the equilibrium shape of the interface between $\mathrm{SO}$ and aqueous for the case of a myopic eye with an axial length of $26.6 \mathrm{~mm}$. This figure is analogous to Figures $5 \mathrm{a}$ and $\mathrm{b}$, i.e., we consider three different volume fractions. In Figure $7 \mathrm{~b}$ we plot the relative tamponated retinal area vs the filling ratio for the case of the emmetropic eye (same curve as in Fig. 7a for SO) and the corresponding curve for a highly myopic eye.

The figure shows that the tamponating effect of SO in the case of the myopic eye is smaller than in the emmetropic one. In fact, the relative tamponated surface $S_{\text {tamponated }} / S$ in the case of SO monotonically decreases with the axial length of the eye, as is shown in Figure 12 (solid circles). On the other hand, for the gas $S_{\text {tamponated }} / S$ remains approximately constant as the axial length of the eye is changed (open circles). This is also the case if a flat configuration of the interface between the tamponade fluid and the aqueous is assumed (solid squares in Fig. 12). 


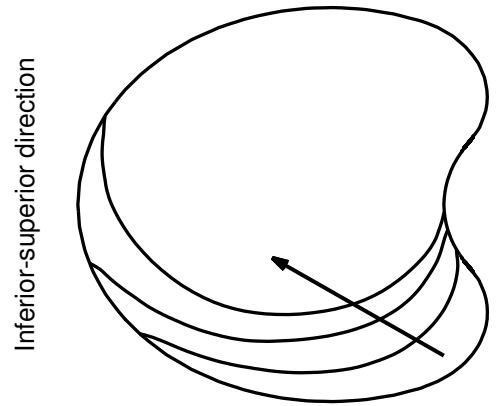

(a)
Posterior-anterior direction

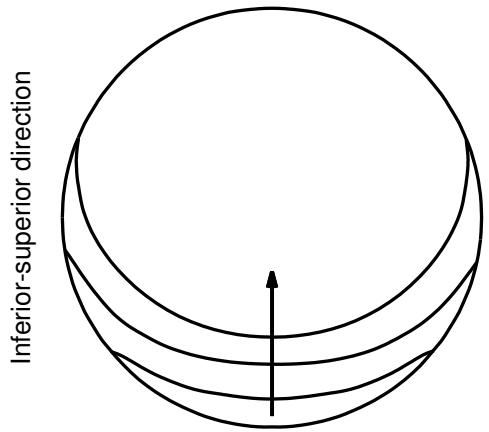

(b)

Nasal-temporal direction

Fig. 11. Equilibrium shapes of the interface in a highly myopic eye for the case of SO. The axial length is $26.6 \mathrm{~mm}$, the height and width are $22.75 \mathrm{~mm}$. The arrows indicate decreasing values of $V_{T . F .} / V$ and the volume fractions are the same as in Fig. 4.

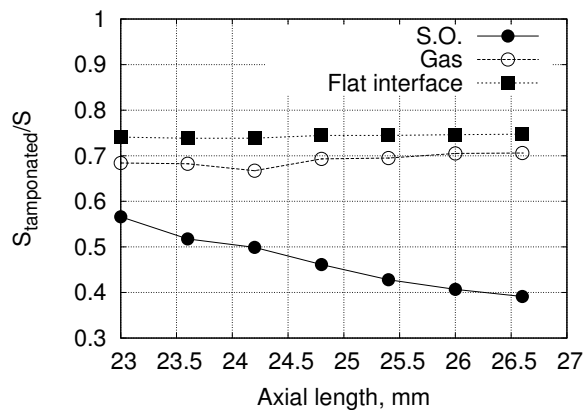

Fig. 12. Relative tamponated surface in the case of $\mathrm{SO}$ as a function of axial length. Filling ratio $V_{T . F .} / V=0.81$. 


\section{Discussion}

Tamponade compounds play an important role in the treatment of retinal detachment. In order to achieve the best possible tamponating effect, the surgeon attempts to fill the vitreous cavity completely with the endotamponades at the end of the surgery. However, under filling is always present due to the hydrophobic properties of the tamponade, which is also affected by the shape of the eyeball and the physical properties of the fluids (mainly their interfacial tension and density). ${ }^{17}$ Thus, the endotamponade is kept away form the retina in certain regions, leaving a variable angle of retina surface without any support. Considering that RBs are often multiple and localized in different quadrants of the retinal surface, it is important for the surgeon to have a better understanding of the shape of the vitreous substitute-aqueous interface, and hence, of its tamponating effect.

Hillier et al. ${ }^{18}$ investigated the influence of axial myopia on tamponade efficacy. The experiments were conducted in vitro using $19 \mathrm{~mm}$ and $25 \mathrm{~mm}$ spherical model chambers to mimic the vitreous cavity. The tamponating efficacy was estimated by measuring the maximum height of the bubble and the arc of contact subtended by the bubble. The authors reported no significant difference in tamponade efficacy according to the size of the eye chamber simulator. The limitation of such a remarkable experiment is mainly the assumption that the vitreous chamber is, in fact, spherical.

In our work, we employed a mathematical model and studied the tamponating effect of two different fluids used during vitrectomy: intraocular gas and SO. We note that the model is based on the solution of well-known equations and the results should be expected to be highly reliable. We considered idealized eye shapes and real eye geometries obtained from MR-images. In addition to this, we also considered idealized eyes with different degrees of myopia.

The results show that, for patients in the upright position, the geometry of the vitreous chamber has a significant impact on the final equilibrium configuration of the interface between the two fluids. This is because the interface intersects the anterior region of the vitreous chamber, where the indentation produced by the lens induces a significant change in the curvature of the vitreous chamber wall. Gas showed better tamponating properties than SO. This is due to two combined effects. First, the contact angle is smaller in the case of SO; second, the density difference is much higher in the case of gas (see Table 1). As a result, the interface with the aqueous is flatter in the case of gas than in the case of SO. The results obtained in the case of a real eye shape reconstructed from MR-images are very close to those obtained in the idealized case.

We also found that, for a given volume fraction of tamponade, the tamponating efficacy of SO is reduced in highly myopic eyes, whereas it remains approximately constant for a gas. This result for SO is interesting and counterintuitive, since one would expect that, in a larger domain (as is the case for a highly myopic eye), the interface should be flatter, thus providing a better tamponating effect. In fact, this is what happens in a spherical domain by increasing the radius, as we have verified with the in-house developed code described in section 2.4. For instance, in the case 
of SO with a filling ratio of 0.80 , the relative tamponated area grows almost linearly from $\approx 0.46$ to $\approx 0.48$ by increasing the sphere radius from 1.0 to $1.5 \mathrm{~cm}$. The finding that, with SO in a myopic eye, the relative tamponated surface decreases with the axial length implies that, in this case, changes in eye shape play a more important role than changes in volume.

Patients who undergo vitrectomy for retinal detachment are asked to keep a certain head position for some time. Typically, after macular hole surgery they are asked to keep their face down because the hole is located in the posterior pole. Very often, however, retinal tears are located in the superior retina due to of gravitational traction forces induced by the vitreous body. In such cases, an upright position is required. In this paper we restricted our attention to this last case, but the method could be easily adopted to treat cases of different head orientations.

Finally, we note that this model can be a clinically valuable tool for eyes with pathological geometries, in which the resulting shape and position of the tamponade compound might differ significantly from the idealized eye shapes, whereby patientspecific evaluation is needed.

\section{Acknowledgements}

The present work was partially carried out at CINECA within the ISCRA C project (HP10CSL8TA).

\section{References}

1. Scott JD. Prevention and perspective in retinal detachment. Eye, 1989;3(5): 491-515.

2. Romano MR, Das R, Groenwald C, Stappler T, Marticorena J, Valldeperas X, et al. Primary 23-gauge sutureless vitrectomy for rhegmatogenous retinal detachment. Indian journal of ophthalmology, 2012;60(1): 29.

3. D’Amico DJ. Primary Retinal Detachment. New England Journal of Medicine, 2008;359(22): PMID: 19038880, 2346-2354. ISSN: 0028-4793. Visited on 02/14/2014, doi: 10.1056/NEJMcp0804591.

4. Oster SF, Mojana F, Bartsch D-UG, Goldbaum M, Freeman WR. Dynamics of the Macular Hole-Silicone Oil Tamponade Interface with Patient Positioning as Imaged by Spectral Domain Optical Coherence Tomography. Retina (Philadelphia, Pa.) 2010;30(6): 924.

5. Fawcett I, Williams R, Wong D. Contact angles of substances used for internal tamponade in retinal detachment surgery. Graefe's archive for clinical and experimental ophthalmology, 1994;232(7): 438444.

6. Eames I, Angunawela RI, Aylward GW, Azarbadegan A. A theoretical model for predicting interfacial relationships of retinal tamponades. Investigative ophthalmology \& visual science, Apr. 2010;51(4): PMID: 19933190, 2243-2247. ISSN: 1552-5783. doi: 10.1167/iovs.09-4442.

7. Pozrikidis C. Fluid Dynamics: Theory, Computation, and Numerical Simulation. Softcover reprint of hardcover 2nd ed. 2009. Springer, Nov. 2010; ISBN: 1441947191.

8. Joussen AM, Wong D. The concept of heavy tamponades-chances and limitations. Graefe's Archive for Clinical and Experimental Ophthalmology, 2008;246(9): 1217-1224.

9. Deshpande SS, Anumolu L, Trujillo MF. Evaluating the performance of the two-phase flow solver interFoam. Computational science \& discovery, 2012;5(1): 014016. 
10. Hirt CW, Nichols BD. Volume of fluid (VOF) method for the dynamics of free boundaries. Journal of computational physics, 1981;39(1): 201-225.

11. OpenFOAM, the Open Source CFD Toolbox by OpenCFD Ltd., Web site available at http://openfoam.com.

12. Atchison DA. Optics of the Human Eye. Elsevier, 2000;

13. Atchison DA, Jones CE, Schmid KL, Pritchard N, Pope JM, Strugnell WE, et al. Eye Shape in Emmetropia and Myopia. Investigative Ophthalmology \& Visual Science, Oct. 2004;45(10): 3380-3386. doi: 10.1167/ iovs.04-0292.

14. Beenakker J, Rijn G, Luyten G, Webb A. High-resolution MRI of uveal melanoma using a microcoil phased array at 7 T. NMR in Biomedicine, 2013;26(12): 1864-1869.

15. Beenakker J-WM, Shamonin DP, Webb AG, Luyten GP, Stoel BC. Automated retinal topographic maps measured with magnetic resonance imagingretinal topographic maps measured with MRI. Investigative ophthalmology \& visual science, 2015;56(2): 1033-1039.

16. Berkowitz BA. MRI of retinal and optic nerve physiology. NMR in Biomedicine, 2008;21(9): 927-927.

17. Wong D, Van Meurs J, Stappler T, Groenewald C, Pearce I, McGalliard J, et al. A pilot study on the use of a perfluorohexyloctane/silicone oil solution as a heavier than water internal tamponade agent. British Journal of Ophthalmology, 2005;89(6): 662-665.

18. Hillier RJ, Stappler T, Williams RL, Turner GS, Wong D. The impact of axial length on retinal tamponade for gas, silicone oil, and heavy silicone oil, using an in vitro model. Graefe's Archive for Clinical and Experimental Ophthalmology, 2011;249(5): 671-675. 